\title{
Variable phenotype in 17q12 microdeletions: Clinical and molecular characterization of a new case
}

\author{
Pietro Palumbo a,b,1, Vincenzo Antona ${ }^{\mathrm{c}, 1}$, Orazio Palumbo ${ }^{\mathrm{a}}$, Maria Piccione ${ }^{\mathrm{c}}$, Rosaria Nardello ${ }^{\mathrm{c}}$, \\ Antonina Fontana ${ }^{c}$, Massimo Carella ${ }^{\mathrm{a}, *}$, Giovanni Corsello ${ }^{\mathrm{c}}$ \\ a Medical Genetics Unit, IRCCS Casa Sollievo della Sofferenza, San Giovanni Rotondo, FG, Italy \\ b Department of Biology, University of Bari, Bari, Italy \\ c Dipartimento di Scienze per la Promozione della Salute e Materno Infantile “G. D'ALESSANDRO”, Universita' Degli Studi di Palermo, Palermo, Italy
}

\section{A R T I C L E I N F O}

\section{Article history:}

Accepted 14 January 2014

Available online 29 January 2014

\section{Keywords:}

HNF1B

$17 \mathrm{q} 12$

SNP array

Renal Cysts and Diabetes syndrome

Intellectual disability

\begin{abstract}
A B S T R A C T
Microdeletions of 17q12 including the hepatocyte nuclear factor 1 beta (HNF1B) gene, as well as point mutations of this gene, are associated with the Renal Cysts and Diabetes syndrome (RCAD, OMIM 137920) and genitourinary alterations. Also, microdeletions encompassing HNF1B were identified as a cause of Mayer-RokitanskyKüster-Hauser Syndrome (MRKH, OMIM 277000) in females and, recently, were associated with intellectual disability, autistic features, cerebral anomaly and facial dysmorphisms.

In this report, we describe a boy with a deletion in 17q12 region detected by SNP array, encompassing the HNF1B gene, that showed dysmorphic features, intellectual disability (ID), serious speech delay and autistic features. In addition, obesity was observed. In order to study the parental origin of the rearrangement, we analyzed selected SNPs in the deleted area in the patient and his parents, showing Mendelian incompatibilities suggesting a de novo deletion on the chromosome of maternal origin.

Our case confirms the incomplete penetrance and variable expressivity of this deletion, its complex clinical variability, and strengthens the evidence that ID and stereotyped behaviors may be part of the phenotypic spectrum characterizing the affected patients. Also, it is useful to further delineate the phenotypes associated to the deletion being the first case in which obesity has been documented. We present a genotype-phenotype correlation discussing the possible role of some genes, encompassed by the deletion, in the etiology of the observed phenotypes.
\end{abstract}

(c) 2014 Elsevier B.V. All rights reserved.

\section{Introduction}

Recurrent genomic rearrangements of chromosome $17 \mathrm{q} 12$ are associated with varied clinical phenotypes. In particular, microdeletions encompassing the hepatocyte nuclear factor 1beta gene (HNF1B, OMIM 189907) are considered the cause of Renal Cysts and Diabetes

Abbreviations: ACACA, Acetyl-CoA Carboxylase Alpha; ADHD, Attention Deficit Hyperactivity Disorder; ASD, Autism Spectrum Disorders; CNTNAP2, Contactin associated protein-like 2; CNV, Copy Number Variation; DNA, Deoxyribonucleic Acid; FOXP2, Forkhead box P2; HNF1B, Hepatocyte Nuclear Factor 1 Beta; ID, Intellectual disability; LCRs, Low Copy Repeats; LHX1, LIM homeobox 1 LIM homeobox 1; MET, Met proto-oncogene; MODY5, Maturity Onset Diabetes of the Young type 5; MRKH, Mayer-Rokitansky-Küster-Hauser-Syndrome; NAHR, Non-Allelic Homologous Recombination; PCR, Polymerase Chain Reaction; RCAD, Renal Cysts and Diabetes syndrome; SNP, Single Nucleotide Polymorphism; TBX1, T-box 1.

* Corresponding author at: Medical Genetics Unit, IRCCS Casa Sollievo della Sofferenza, 71013 San Giovanni Rotondo, FG, Italy. Tel.: + 390882 416350; fax: + 390882411616.

E-mail address: m.carella@operapadrepio.it (M. Carella).

${ }^{1}$ Equal contributors.
(RCAD) syndrome, an autosomal dominant disorder (Bingham et al., 2004). This condition results in a clinically distinguishable phenotype, whose major features include cystic renal disease, renal anomaly, maturity onset diabetes of the young type 5 (MODY5), pancreatic atrophy, and liver abnormalities (Bellanné-Chantelot, 2005; Nagamani et al., 2010).

Also, deletions in $17 q 12$ have recently been identified as a rare cause of Mayer-Rokitansky-Küster-Hauser Syndrome (MRKH). This syndrome is defined in 46,XX women by congenital absence of the uterus and upper part of the vagina with otherwise normal sexual development and can be associated with renal, skeletal and/or hearing defects (Bernardini et al., 2009; Nik-Zainal et al., 2011 The 17q12 microdeletions are not characterized by intellectual disability or other neurobehavioral phenotypes, while it was observed that the duplication of the same region causes intellectual disability, autism spectrum disorder and speech delay (Brandt et al., 2012). Although earlier reports of cases with the $17 q 12$ microdeletion syndrome did not include cognitive impairment as a part of the characteristic phenotype of these deletions (Mefford et al., 2007), recently, some authors (Dixit et al., 2012; Loirat et al., 2010; Mukamel et al., 2011) 
have reported rare cases with intellectual impairment, with or without autism, suggesting that cognitive impairment and a behavioral phenotype could be part of the clinical features conveyed by the $17 q 12$ microdeletion. In order to deeply investigate the phenotype associated with $17 q 12$ microdeletion, we describe the clinical and molecular data of a patient carrier of a de novo deletion in the $17 q 12$ region, $1.42 \mathrm{Mb}$ in size, with dysmorphic features, serious speech delay, intellectual disability (ID), stereotyped behaviors and obesity, but without the typical clinical features usually present in patients harboring this deletion. Finally, we discuss candidate genes for the observed traits and, in particular, we discuss the possible role of involvement of MIR2929 in the del17q12 neurological phenotype observed in our patient.

\section{Clinical description}

The patient is a 12-year-old boy whose parents are nonconsanguineous. There is no family history of ID, autism, or other psychiatric illness. He was born at 41 weeks of gestation by cesarean section. The child had been naturally conceived and the parents were not aware of any abnormalities on prenatal ultrasounds. At birth an echocardiographic exam was performed, which showed transposition of great arteries, surgically treated at 40 days of life.

Developmental milestones were normal until 14 months. In the language domain, the first use of single words by the patient occurred at 5 years without further maturation of verbal communication skills, and currently he has a vocabulary of a few words.

At a physical examination at the age of 11 years, his weight was $62 \mathrm{~kg}$ (>97th centile), his height was $152 \mathrm{~cm}$ (>75th centile), and his head circumference measured $52 \mathrm{~cm}(<10$ th centile). He was engaging in repetitive and compulsive-like behaviors. The patient showed attention deficit hyperactivity disorder (ADHD) and intellectual disability.

He expressed himself in language characterized by vocalizations with communicative value and he was even able to answer simple deliveries. The patient kept the static standing with plantigrade support and with evident motor clumsiness during the gait pattern. The manipulative skills were characterized by digitopalmar prehension with greater intentionality to the right and lack of integration into fine motor activities. Neurological exams showed normal cranial nerves, normal muscle tone and trophism, normal ROT symmetrical in all four limbs and no evidence of stenic deficits or pyramidal signs.

On physical examination the patient showed dysmorphic features in the form of right posterior plagiocephaly, facial asymmetry, narrow forehead, hypotelorism, wide and fleshy auricular pavilions, protruding cheekbones, long philtrum, thin upper lip, tuft of hair on the neck to the left parotid region, clinodactyly of the fifth fingers of the hands (Supplementary Fig. 1).

A full ultrasound abdomen exam showed regular liver morfovolumetry and echotexture, normal gallbladder, not dilated intra-and extrahepatic bile ducts, portal vein caliber regular hilum, regular spleen echotexture and morphovolumetry. Kidney's echotexture and morfovolumetry were regular and without signs of hydronephrosis, bladder was free of endoluminal alterations.

\section{Matherials and methods}

\subsection{Snp array assay}

Blood was obtained from the proband and his parents after signed informed consent. The study was designed to perform parental and proband samples concurrently on three individual chips. Genomic DNA was isolated from peripheral blood lymphocytes of the patient and the parents by using BioRobot EZ1 (Quiagen, Solna, Sweden). DNA concentration and purity were determined with a ND-1000 Spectrophotometer (NanoDrop Technologies, Berlin, Germany) while SNP-array analysis was performed by using Affymetrix GeneChip Genome Wide Human SNP 6.0 Array (Affymetrix, Santa Clara, CA). This array has, on average, an inter-marker distance of 700 bp and makes possible an excellent assessment of possible breakpoints of deletions to assess copy gains or losses. The SNP 6.0 assay was performed according to the manufacturer's protocol, starting with $500 \mathrm{ng}$ of DNA. Briefly: total genomic DNA was digested with restriction enzymes (NspI and StyI), ligated to appropriate adapters for the enzymes, and subjected to PCR amplification using a single primer. After digestion with DNase I, the PCR products were labeled with a biotinylated nucleotide analog using terminal deoxynucleotidyl transferase and hybridized to the microarray. Hybridization was carried out in the Affymetrix Hybridization Owen 450 while subsequent washing and staining steps were performed using the Fluidic Station 450 and finally the array was scanned with the GeneChip Scanner 3000 7G using Command Console Software (Affymetrix, Santa Clara, CA, USA).

Analysis was performed using Genotyping Console Software 4.1 (Affymetrix, Santa Clara, CA, USA) and Birdseed v2-algorithm. Samples were normalized against 270 International HapMap samples (Affymetrix, CA) while an additional 80 in-house control samples, which were hybridized in our facility, were used to decrease technical variation. For the copy number analysis, we used regional GC correction and required 25 markers to be found within the changed region and the size of the region to be at least $200 \mathrm{~kb}$. The amplified and/or deleted regions were detected using a standard Hidden Markov Model (HMM) method. Genotyping Console Browser (Affymetrix, CA) was used to illustrate changes detected while the University of California Santa Cruz (UCSC) Genome Browser (http://genome.ucsc.edu/cgi-bin/ hgGateway), assembly GRCh37, was used to map the genomic coordinates and find the genes within the copy number altered region.

\subsection{Haplotype analysis}

A total of 23 informative single nucleotide polymorphisms (SNPs) were selected from 1827 SNPs located at 17q12 (physical position 31.8-38.1 Mb) for haplotype structural analysis. Phased haplotypes were inferred from genotype data in both parents and child. The 17 SNPs (from rs9912731, physical position 35,239,742 bp, to rs8069122, physical position 36,211,659 bp) showed inconsistencies of Mendelian inheritance, marking hemizygous genotypes of these SNPs being miscalled as homozygous.

\subsection{Mutational analysis}

To amplify the entire coding sequence and flanking intronic portions of $L H X 1,5$ primer pairs were designed. Mutational analysis of $L H X 1$ gene has been performed as already reported in Bernardini et al. (Bernardini et al., 2009).

To amplify the entire coding sequence and flanking intronic portions of ACACA, 53 primer pairs were designed (Supplementary Table 1). The PCR reactions (except exon 45) were performed in a $25 \mu \mathrm{L}$ volume containing $1.5 \mathrm{mM} \mathrm{MgCl} 2,250 \mu \mathrm{M}$ dNTPs, $1 \mu \mathrm{M}$ of each primer, $50 \mathrm{ng}$ of genomic DNA and $1.5 \mathrm{U}$ of AmpliTaq Gold DNA polymerase (Applied Biosystems, Foster City, CA) with an initial denaturation step of $12 \mathrm{~min}$ at $95{ }^{\circ} \mathrm{C}$ followed by 35 cycles at $94{ }^{\circ} \mathrm{C}, 30 \mathrm{~s} ; 60{ }^{\circ} \mathrm{C}, 30 \mathrm{~s} ; 72{ }^{\circ} \mathrm{C}$, $30 \mathrm{~s}$ and a final elongation of $7 \mathrm{~min}$ at $72{ }^{\circ} \mathrm{C}$. Exons $27,34-35$ were amplified with an initial denaturation step of $12 \mathrm{~min}$ at $95^{\circ} \mathrm{C}$ followed by 35 cycles at $94{ }^{\circ} \mathrm{C}, 30 \mathrm{~s} ; 58{ }^{\circ} \mathrm{C}, 30 \mathrm{~s} ; 72^{\circ} \mathrm{C}, 30 \mathrm{~s}$ and a final elongation of 7 min at $72{ }^{\circ} \mathrm{C}$. ACACA exon 45 has been amplified in a $25 \mu \mathrm{l}$ volume containing $5 \mu \mathrm{L}$ of $5 \times$ GC-Rich solution (Roche Applied Science, Mannheim, Germany), a $2.5 \mu \mathrm{L}$ of $10 \times$ FastStart Taq DNA Plymerase Buffer (Roche Applied Science, Mannheim, Germany), $250 \mu \mathrm{M}$ dNTPs, $1 \mu \mathrm{M}$ of each primer, 50 ng of genomic DNA and 2 U of FastStart Taq DNA Plymerase (Roche Applied Science, Mannheim, Germany) with an initial denaturation step of $3 \mathrm{~min}$ at $95{ }^{\circ} \mathrm{C}$ followed by 35 cycles at $94{ }^{\circ} \mathrm{C}, 30 \mathrm{~s}$; $58{ }^{\circ} \mathrm{C}, 30 \mathrm{~s} ; 72{ }^{\circ} \mathrm{C}, 30 \mathrm{~s}$ and a final elongation of $7 \mathrm{~min}$ at $72{ }^{\circ} \mathrm{C}$. All PCR products were purified using ExoSAPIT (USB Corporation, Cleveland, $\mathrm{OH}$ ) and sequenced directly on an automated sequencer (ABI 3100; 


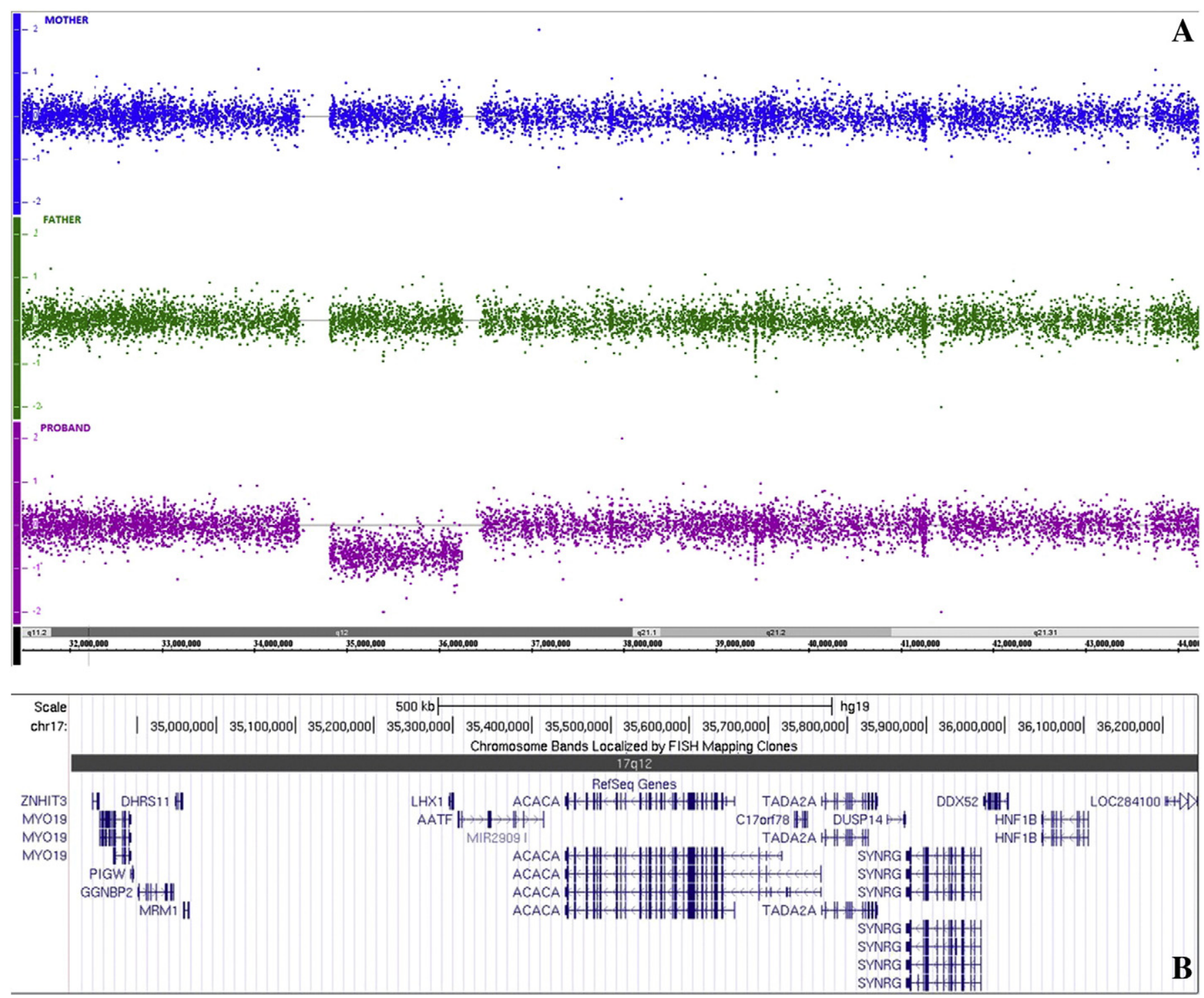

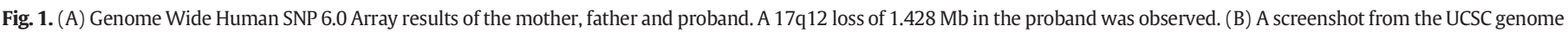
browser showing the deleted region at cytogenetic location 17q12, from 34,816,256 to 36,244,358 bp, based on the Human Genome February 2009 (GRCh37/hg19) assembly.

Applied Biosystems, Foster City, CA) using the ABI-PRISM big-dye Terminator Cycle Sequencing Ready Reaction kit (Applied Biosystems, Foster City, CA).

\section{Results}

SNP-array analysis of the proband revealed a heterozygous deletion involving interstitial chromosome 17q12, from SNP_A-8624766 $(34,816,256$ bp) to SNP_A-1828153 (36,244,358 bp; rs1963088) (USCS Genome Browser build February 2009 hg19), which were the first and the last deleted oligonucleotide from the centromere respectively. The flanking normal probes were CN_754222 (34,629,684 bp) and CN_763010 (36,282,179 bp). The subsequent microarray analysis of the proband's parents using the same platform revealed normal chromosomes 17 in both of them, indicating a de novo deletion in the child (Fig. 1A). The minimum size of loss was $1.428 \mathrm{Mb}$, covered by 973 SNP array probes, while the maximum size of deletion was $1.652 \mathrm{Mb}$. The molecular karyotype of the patient was arr17q12 $(31,500,383 \times 2$, $34,816,255-36,244,358 \times 1,36,300,595 \times 2) d n$. The hemizygous region included the entire coding sequence of 16 genes (CCL4L2, TBC1D3H,
TBC1D3G, ZNHIT3, MYO19, PIGW, GGNBP2, DHRS11, MRM, LHX1, AATF, ACACA, TADA2A, DUSP14, SYNRG, HNF1B) and a miRNA (mir-2909) (Fig. 1B). The subsequent genotype analysis showed that the deletion affects the maternal allele (Fig. 2). The deletion in the patient, as well as the parental origin, were confirmed by a second experiment using the CytoScan HD array (Affymetrix, Santa Clara, CA) which contains more than 2.6 million markers for copy number analysis and approximately 750,000 SNPs that fully genotype with greater than 99\% accuracy. A loss of $1.421 \mathrm{Mb}$ was identified at $17 \mathrm{q} 12$, covered by 1652 SNP array probes (Supplementary Fig. 2). The proximal breakpoint (centromeric) was located between the last present probe S-4ESPG (34,477,480 bp; rs916841) and the first deleted probe C-6IWKL (34,822,466 bp), while the distal breakpoint (telomeric) was located between the last deleted probe S-4AOXU (36,244,358 bp; rs1963088) and the first present probe C-3ZZGO $(36,283,612 \mathrm{bp})$. The telomeric end point of the deletion identified is the same in both the analysis while the centromeric end point is quite different due to the slightly different distribution of the probes on the two arrays. It is important to note that this difference in size does not modify the gene content of the reported deletion. 


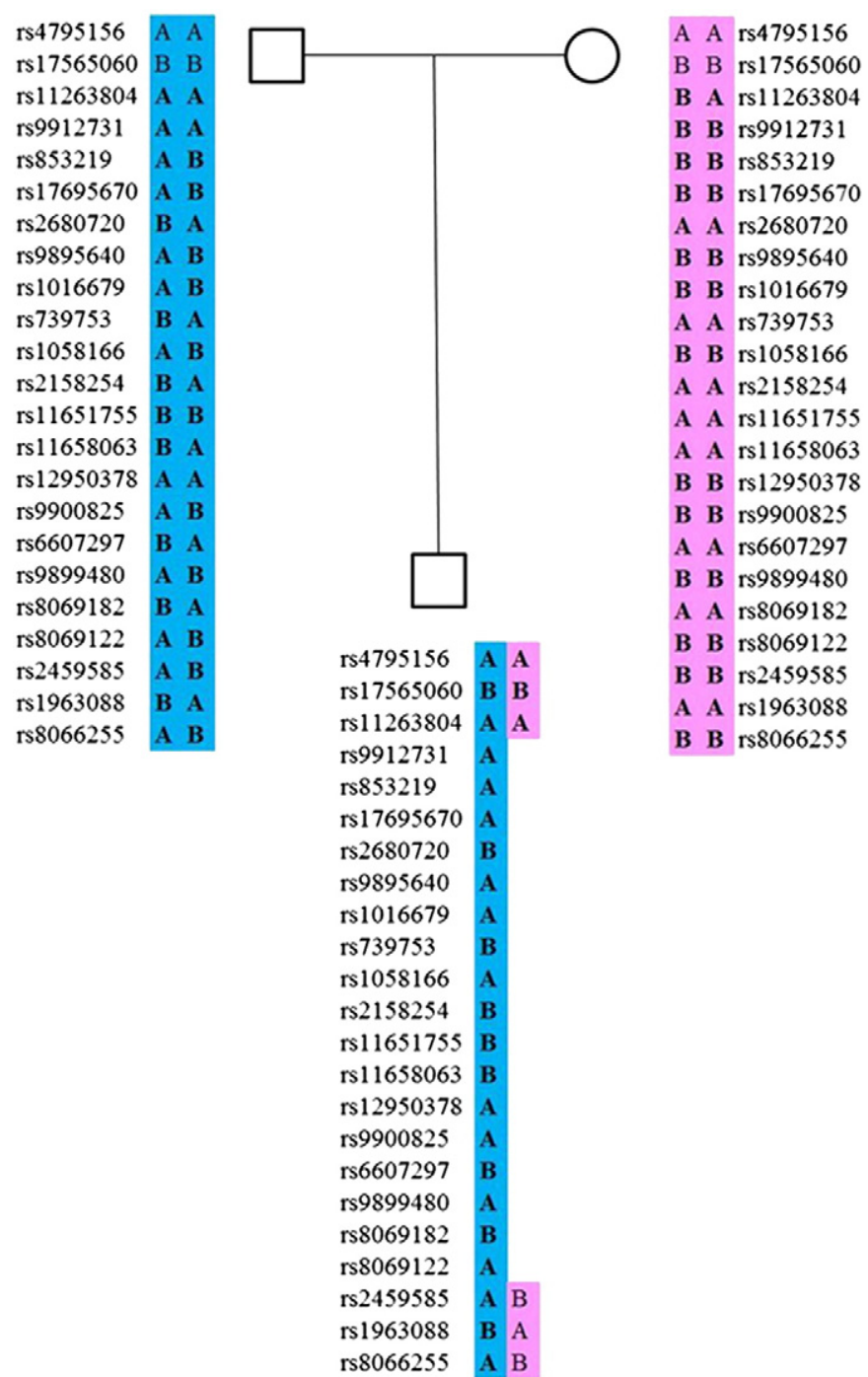

Fig. 2. Results of haplotype analysis. The phased haplotypes based upon 23 selected informative SNPs from the centromeric, the deleted and the telomeric regions are reported. Hemizygous calls instead of homozygous calls of SNPs within the deleted region are shown since loss of one copy in the proband is confirmed. The marked markers are informative SNPs to demonstrate maternal deletion event of the region.

After direct sequencing, no pathogenic mutations or polymorphisms were found in $L H X 1$ gene, while in ACACA gene we find a polymorphism in exon 50 (T2063T).

\section{Discussion}

The 17q12 deletion, involving HNFB1 gene, is associated with the RCAD (renal cysts and diabetes) syndrome, characterized by cystic renal disease, renal anomaly, MODY5, pancreatic atrophy and liver abnormalities. In some cases the same deletion is associated with MRHK (Mayer-Rokitansky-Küster-Hauser) syndrome, characterized by congenital absence of the uterus and upper part of vagina with otherwise normal sexual development in 46,XX women and can be associated with renal, skeletal and/or hearing defects. The 17q12 deletion was the first genomic disorder resulting in diabetes and was the third genomic disorder with significant renal involvement (Mefford et al., 2007). Chen et al. (2010)) reviewed several cases of HNF1B anomalies (especially mutations) in renal cysts and diabetes syndrome/maturity onset diabetes of the young type 5 , deducting that renal structure anomalies (RSA) represented the most frequent phenotype (89.6\%) while diabetes mellitus (DM) was $45 \%$.
The majority of $17 q 12$ deletion cases are intellectually normal. Despite it was observed that the duplication of the same region causes intellectual disability, autism spectrum disorder and speech delay (Brandt et al., 2012), first reports of 17q12 microdeletions did not associate the rearrangement with any form of intellectual disability and/or autism, suggested that there may also be a correlation between $17 \mathrm{q} 12$ microdeletions and intellectual disabilities/autism, but these features do not seem to be completely penetrant. Moreno-De Luca and SGENE Consortium (2010) demonstrated that 17q12 deletion, in addition to causing kidney disease, is a recurrent pathogenic CNV that confers risk for ASD because may involve dosage sensitive genes essential for normal brain development. Loirat et al. (Loirat et al., 2010) reported three patients with autism, cystic or hyperechogenic kidney carriers of a17q12 heterozygous deletion encompassing HNF1B. Then, Nagamani et al. (Nagamani et al., 2010) reported three cases with deletion in $17 q 12$ presented with developmental delay/cognitive impairment. Recently, two additional patients have been reported by Dixit et al. (Dixit et al., 2012), with variable neurocognitive involvement. Very interesting, rarely dysmorphic features for these patients have been documented (Bernardini et al., 2009; Moreno-De Luca and SGENE Consortium, 2010). Thus, with the evidence reported to date, the 17q12 deletions seem to be responsible for a wide range of phenotypes with considerable variability in expressivity and penetrance.

In this report we describe a boy with a deletion in $17 q 12$ region detected by SNP array, that showed dysmorphic features (right posterior plagiocephaly, facial asymmetry, narrow forehead, hypotelorism, wide and fleshy auricular pavilions, protruding cheekbones, long philtrum, thin upper lip, tuft of hair on the neck to the left parotid region, clinodactyly of the fifth fingers of the hands), ID, serious speech delay and autistic features. In addition, obesity was observed. Clinical comparison between our patient and the others previously described is reported in Table 1.

The minimum size of loss was $1.428 \mathrm{Mb}$ while the maximum size of deletion was $1.652 \mathrm{Mb}$. Since both the proximal and the distal breakpoints coincided with published DNA copy number polymorphisms and were found enriched for low-copy repeats (LCRs) (Bellanné-Chantelot, 2005), the mechanism of the deletion was probably a non-allelic homologous recombination (NAHR) (Mefford et al., 2009). Also, some authors reported cases carrying the reciprocal duplication of the same region (Mefford et al., 2007) providing further evidence that these rearrangements are mediated by NAHR. The hemizygous region in our patient included 16 known genes and a miRNA and, although the 17q12 deletion genotype/phenotype correlation is extremely variable suggesting an incomplete penetrance and variable expressivity, haploinsufficiency of some of them might explain the phenotype observed in our patient.

Genetic causes for autism and/or intellectual disability in patients with $17 q 12$ deletion are uncertain. Deletion size and breakpoint locations in our patient with neurobehavioral phenotype, and others reported with intellectual disability, are similar to those reported in patients without these clinical features. Thus, the co-occurrence of multiple, apparently unrelated, clinical findings in patients with the 17q12 deletion, is suggestive of a contiguous gene syndrome. Under this model, major phenotypic features may be due to haploinsufficiency of two or more different genes within the deletion. HNF1B is responsible for the renal structural abnormalities since mutations in this gene cause RCAD syndrome. There are no reported neurocognitive or psychiatric phenotypes in patients with RCAD resulting from point mutations in $H N F 1 B$, although this may not have been assessed in a systematic fashion. If patients with HNF1B mutations do not show neurobehavioral phenotype, a different gene within the deleted region may account for this component of the phenotype. Point mutations in this other gene may also occur in patients with a neurobehavioral impairment who do not have the renal and endocrine phenotype due to HNF1B haploinsufficiency. Of 16 genes involved in the microdeletion, in agreement with other authors, we consider LHX1 as a serious candidate to explain the 
Table 1

Clinical phenotype in patients with 17q12 deletions.

\begin{tabular}{|c|c|c|c|c|c|c|c|c|c|c|c|c|}
\hline \multirow[b]{2}{*}{ Age of diagnosis } & \multirow{2}{*}{$\begin{array}{l}\text { Present case } \\
12 \text { years }\end{array}$} & \multicolumn{3}{|c|}{ Dixit et al. (2012) } & \multicolumn{4}{|c|}{ Nagamani et al. (2010) } & \multirow{2}{*}{$\begin{array}{l}\text { Moreno-De Luca and } \\
\text { SGENE Consortium (2010) }\end{array}$} & \multicolumn{3}{|c|}{ Loirat et al. (2010) } \\
\hline & & 12 years & 4 years & 7 months & 5 years & 4 months & 13 years & 18 years & & 9 years & 4 years & 3 years \\
\hline $\begin{array}{l}\text { Developmental delay/cognitive } \\
\text { impairment }\end{array}$ & + & + & + & NA & + & - & + & + & $8 / 9$ & + & + & + \\
\hline Seizures & - & - & - & & - & - & + & + & - & NA & NA & NA \\
\hline Structural brain anomalies & - & - & NA & + & - & - & + & + & NA & + & + & NA \\
\hline Dysmorphic features & + & - & + & + & + & - & - & - & $8 / 9$ & NA & NA & NA \\
\hline Renal cysts & - & + & + & + & + & + & - & + & $9 / 9$ & + & + & + \\
\hline Renal abnormalities & - & + & + & - & + & - & - & + & $8 / 9$ & + & + & + \\
\hline Obesity & + & - & - & - & - & - & - & - & NA & NA & NA & NA \\
\hline Diabetes & - & NA & NA & NA & NA & NA & NA & + & $1 / 9$ & NA & NA & NA \\
\hline
\end{tabular}

+, present; -, absent; NA, not available.

neurobehavioral phenotype observed in our patient. As a potential transcriptional regulator that plays a role in the differentiation of neural cells and the transcriptional control of axonal guidance, it is expressed in the brain during early development (Avraham et al., 2009). Furthermore, an Lhx1 knockout mouse model exhibits anencephaly, showing that LHX1 is an essential regulator of the vertebrate head organizer (Shawlot et al., 1995). LHX1 has yet to be associated with disease in humans, and no single gene deletions, disruptions, or mutations causing haploinsufficiency have been detected. Contiguous gene disorders, such as the 16p11.2 deletion syndrome (OMIM 611913), also show considerable variability (Ballif et al., 2007), with a "second hit" possibly affecting severity. We sequenced LHX1 and we didn't find any mutations in this gene. This result strengthens our hypothesis according to which haploinsufficiency of $L H X 1$ could be the mechanism involved in the clinical feature. Probably, being $L H X 1$ a transcription factor strongly involved in early development of central nervous system, human embryos with a total absence of $L H X 1$ may be non-viable. We can therefore hypothesize that the deletion of $L H X 1$ is a necessary but not sufficient condition for arising intellectual disability. This second event could be a smaller CNV occurring elsewhere in the genome, a small disruptive mutation in a related gene, an environmental event leading to alteration in gene dosage. Future studies are therefore necessary to identify the gene or genes responsible for the neurobehavioral phenotype in patients with the $17 \mathrm{q} 12$ deletion.

Interestingly, a non-coding RNA, miR-2909, is located within the deleted region. Several reports indicated a role for non-coding RNAs in the molecular pathogenesis of different developmental disorders (Szulwach et al., 2009). We searched the miRBase database (http:// mirdb.org/miRDB/) for mRNAs containing a putative miR-2909 binding site. This analysis revealed a large number of putative miR-2909 targets mRNAs (Supplementary Table 2). Among these mRNAs, the highest score was reached from FOXP2, which could explain the speech delay and the cognitive/behavioral phenotype observed in our patient. FOXP2 codes for a protein, expressed widely in the fetal and adult brain, where it regulates the expression of several genes within and among cortical, basal ganglia and cerebellar circuits (Vernes et al., 2009). Previous studies demonstrated that FOXP2 is responsible for speech-language disorders and cognitive impairment. To date, disruptions in FOXP2 are the only known monogenic cause of speech and language impairment (Bacon et al., 2012; Benítez-Burraco, 2012. Furthermore, it has been shown that FOXP2 directly regulates CNTNAP2, whose mutations, like FOXP2, were found to be correlated to language impairment (Vernes et al., 2008), and the autism candidate gene MET, involved in neuronal differentiation (Mukamel et al., 2011). Although we have no experimental evidence, we suppose that alterations of its expression pattern could be causative or co-responsible for speech deficit and neurobehavioral features in our patient. Our hypothesis is that, being miRNAs negative regulators of gene expression, the deletion of one of these non-coding RNAs could cause an altered expression of target genes. This hypothesis is compatible with what is already documented in some microdeletion syndromes, for example in 22q11.2 (Xu et al., 2013) and with what has been observed in cases of 17q12 duplication, where over-expression of miR-2909 could cause the decrease of the levels of FOXP2 expression: in fact several studies described deletions of this gene as responsible for language and cognitive disorders. It is widely accepted that, deletion and duplication of dose sensitive genes, as in 22q11 deletions/duplication, may cause a similar phenotype: mice over- and underexpressing TBX1 result in phenotypes similar to that of velocardiofacial syndrome (VCFS) (Liao et al., 2004). Thus we suggest that FOXP2 could be characterized by a similar mechanism, and therefore speech delay and cognitive impairment observed in our patient may be caused by miR-2909 deletion. Obviously, to confirm this suggestive hypothesis, functional or animal studies are needed.

It is important to consider the fact that the neurological phenotype, rather than rare, may simply be underestimated as the carriers of the $17 q 12$ deletion are typically characterized by morphological and/or functional alteration in the kidney and/or diabetes, and therefore followed by specialists who could not take into account variable expressions of autistic features and intellectual disabilities. We suggest the investigation of the presence of autistic features and intellectual disabilities in a large cohort of cases-carriers of 17q12 deletion to corroborate our hypothesis and report the incidence of these phenotypes in patients with the deletion. Also, in our patient the parental origin of the deletion has been investigated and it occurred in the maternal allele, thus we cannot exclude that the phenotypic variability could also be caused by the parental origin of the rearrangement. Therefore we suggest the investigation of the parental origin of the rearrangement, in order to confirm or disprove the possibility that the parental origin of the rearrangement can somehow influence the onset of neurobehavioral phenotype. This systematic analysis could report with more accuracy the incidence of these phenotypes in patients with the 17q12 microdeletions. About the absence of the renal alterations in our patient, unlike what emerged so far, we suggest that not only the neurobehavioral phenotype is subject to incomplete penetrance or variable expressivity, but also the renal phenotype, even if this shows higher penetrance. Moreover, although diabetes is not yet present in the patient, it is not possible to rule out its future occurrence, while the renal phenotype typically has a very early onset and thus should not occur at all.

Regarding the obesity reported in our patient, we focused our attention on the rule of ACACA (Acetyl-CoA carboxylase alpha) gene. AcetylCoA carboxylase (ACC) is a complex multifunctional enzyme system, which catalyzes the carboxylation of acetyl-CoA to malonyl-CoA, the rate-limiting step in fatty acid synthesis. There are two ACC forms, alpha and beta, encoded by two different genes. ACC-alpha is highly enriched in lipogenic tissues, and it has been reported (Jie et al., 2010) that genes related to fatty acid synthesis, including ACACA gene, had decreased expression in obese rats. We sequenced ACACA gene and we didn't find any mutation. It is not yet clear which mechanism is involved, but the deletion of this gene may be responsible for or contributed to the obesity observed in our patient. Obviously further studies 
are needed. This could confirm the high variable expressivity that characterizes this copy number variation, since this is the first patient that shows this feature. Also, we cannot exclude the existence of a position effect on the genes located in the adjacent regions of the deletion as reported in other genomic disorders.

In conclusion, we have described a case of a $17 \mathrm{q} 12$ de novo deletion, detected by SNP array, encompassing the HNF1B gene, in a patient without renal cysts or diabetes, that shows obesity, intellectual disability, serious speech delay and autistic features. Autism and ID should be considered as manifestations associated with 17q12 microdeletions, in particular with LHX1/mir2909 deletion, and should be investigated in a large cohort of patients with 17q12 anomalies to confirm our observations and document the exact incidence of ID in patients harboring this deletion. Furthermore, we highlight that, differently from what has been observed so far, renal phenotype could also be subject to incomplete penetrance and variable expressivity, although to a lesser extent compared to the neurobehavioral phenotype. Our patient is also useful to further expand the clinical spectrum of this microdeletion being the first one in which evident obesity has been reported and we discuss the possible role of a candidate gene in the etiology of this trait.

Supplementary data to this article can be found online at http://dx. doi.org/10.1016/j.gene.2014.01.050.

\section{Conflict of interest}

The authors declare that they have no competing interests.

\section{Acknowledgments}

This study was supported by a grant from the Italian Ministry of Health (Ricerca Corrente 2013) and by the " $5 \times 1000$ " voluntary contributions to MC. We would like to thank the patient and his family for their cooperation.

\section{References}

Avraham, O., et al., 2009. Transcriptional control of axonal guidance and sorting in dorsal interneurons by the Lim-HD proteins Lhx9 and Lhx1. Neural Dev. 4, 21.

Bacon, C., et al., 2012. The distinct and overlapping phenotypic spectra of FOXP1 and FOXP2 in cognitive disorders. Hum. Genet. 131, 1687-1698.
Ballif, B.C., et al., 2007. Discovery of a previously unrecognized microdeletion syndrome of 16p11.2-p12.2. Nat. Genet. 9, 1071-1073.

Bellanné-Chantelot, C., 2005. Large genomic rearrangements in the hepatocyte nuclear factor-1_ (TCF2) gene are the most frequent cause of maturity-onset diabetes of the young type 5 . Diabetes 54, 3126-3132.

Benítez-Burraco, A., 2012. Problematic aspects of the genetic analysis of the specific disorders of the language: FOXP2 as paradigm. Neurologia 27, 225-233.

Bernardini, L, et al, 2009. Recurrent microdeletion at 17q12 as a cause of MayerRokitansky-Kuster-Hauser (MRKH) syndrome: two case reports. Orphanet J. Rare Dis. 4 (4), 25.

Bingham, C., et al., 2004. Renal cysts and diabetes syndrome resulting from mutations in hepatocyte nuclear factor-1beta. Nephrol. Dial. Transplant. 19, 2703-2708.

Brandt, T., et al., 2012. Complex autism spectrum disorder in a patient with a 17q12 microduplication. Am. J. Med. Genet. 158, 1170-1177.

Chen, Y.Z., et al., 2010. Systematic review of TCF2 anomalies in renal cysts and diabetes syndrome/maturity onset diabetes of the young type 5. Chin. Med. J. 123, 3326-3333.

Dixit, A., et al., 2012. 17q12 microdeletion syndrome: three patients illustrating the phenotypic spectrum. Am. J. Med. Genet. 158, 2317-2321.

Jie, Q., et al., 2010. Gene expression profiles of adipose tissue of high-fat diet-induced obese rats by cDNA microarrays. Mol. Biol. Rep. 37, 3691-3695.

Liao, J., et al., 2004. Full spectrum of malformations in velocardio- facial syndrome/ DiGeorge syndrome mouse models by altering Tbx1 dosage. Hum. Mol. Genet. 13, 1577-1585.

Loirat, C., et al., 2010. Autism in three patients with cystic or hyperechogenic kidneys and chromosome 17q12 deletion. Nephrol. Dial. Transplant. 25, 3430-3433.

Mefford, H.C., et al., 2007. Recurrent reciprocal genomic rearrangements of 17q12 are associated with renal disease, diabetes, and epilepsy. Am. J. Hum. Genet. 81 1057-1069.

Mefford, H.C., et al., 2009. Duplication hotspots, rare genomic disorders, and common disease. Curr. Opin. Genet. Dev. 19, 196-204.

Moreno-De Luca, D., SGENE Consortium, 2010. Deletion 17q12 is a recurrent copy number variant that confers high risk of autism and schizophrenia. Am. J. Hum. Genet. 87, 618-630.

Mukamel, Z., et al., 2011. Regulation of MET by FOXP2, genes implicated in higher cognitive dysfunction and autism risk. J. Neurosci. 31, 11437-11442.

Nagamani, S.C., et al., 2010. Clinical spectrum associated with recurrent genomic rearrangements in chromosome 17q12. Eur. J. Hum. Genet. 18, 278-284.

Nik-Zainal, S., et al., 2011. High incidence of recurrent copy number variants in patients with isolated and syndromic Müllerian aplasia. J. Med. Genet. 48, 197-204.

Shawlot, W., et al., 1995. Requirement for Lim1 in head-organizer function. Nature 374, $425-430$.

Szulwach, K.E., et al., 2009. Noncoding RNAs in mental retardation. Clin. Genet. 75, 209-219.

Vernes, S.C., et al., 2008. A functional genetic link between distinct developmental language disorders. N. Engl. J. Med. 22, 2337-2345.

Vernes, S.C., et al., 2009. Unravelling neurogenetic networks implicated in developmental language disorders. Biochem. Soc. Trans. 37, 1263-1269.

Xu, B., et al., 2013. Derepression of a neuronal inhibitor due to miRNA dysregulation in a schizophrenia-related microdeletion. Cell 152, 262-275. 